\title{
ASYMPTOTICS OF COLUMNS IN THE TABLE OF ORTHOGONAL POLYNOMIALS WITH VARYING MEASURES
}

\begin{abstract}
$\mathrm{Xin} \mathrm{Li}$
ABstract. For a given sequence of positive Borel measures $\left\{\mu_{k}\right\}_{k=1}^{\infty}$ in the complex plane $\mathbf{C}$ and $p>0$, we study the asymptotic behavior of the sequence of the $n$th degree monic polynomials with minimal $L_{p}\left(d \mu_{k}\right)$ norm as $k \rightarrow \infty$. Applications to the frequency analysis problem and orthogonal polynomials are discussed. In particular, a " $K_{n}$-result" is established for the $R$-process proposed in the recent work by Jones, Njåstad, and Waadeland [Continued Fractions and Orthogonal Functions, Marcel Dekker, New York, 1994, pp. 141-152].
\end{abstract}

\section{Introduction}

Let $\mathcal{M}$ be the set of all finite positive Borel measures in the complex plane $\mathbf{C}$, and let $\Phi$ denote the set of all continuous functions defined in $\mathbf{C}$ with compact support. We impose on $\mathcal{M}$ the weak star topology using $\Phi$. More precisely, the convergence of a sequence of measures $\left\{\mu_{k}\right\}_{k=1}^{\infty}$ to the measure $\mu$ means

$$
\lim _{k \rightarrow \infty} \int f d \mu_{k}=\int f d \mu
$$

holds for all $f \in \Phi$. The weak star convergence will be denoted by $\mu_{k} \stackrel{*}{\longrightarrow} \mu$.

In this paper, all measures are assumed to be in $\mathcal{M}$. The support of a measure $\mu$ will be denoted by $\operatorname{supp}(\mu)$. Let $\mathcal{N}:=\{0, \pm 1, \pm 2, \pm 3, \ldots\}$ and $\mathcal{N}^{+}:=\{1,2,3, \ldots\}$.

For measure $\mu$, assume that $\operatorname{supp}(\mu)$ is compact. Let $n \in \mathcal{N}^{+}$and $p>0$. Then an $L_{p}(\mu)$-extremal (monic) polynomial of degree $n$, denoted by $P_{n, p}(z ; \mu)=z^{n}+\cdots \in \mathcal{P}_{n}$ (where $\mathcal{P}_{n}$ is the set of polynomials of degree at most $n$ ), is a solution of the following extremal problem:

$$
\int\left|P_{n, p}(z ; \mu)\right|^{p} d \mu=\inf _{\substack{q_{n} \in \mathcal{P}_{n} \\ q_{n}(z)=z^{n}+\cdots}} \int\left|q_{n}(z)\right|^{p} d \mu .
$$

It is well known that when $p>1$ and $\operatorname{supp}(\mu)$ contains infinitely many points, such $L_{p}(\mu)$-extremal polynomial is unique for every $n=1,2, \ldots$ If $1 \geq p>0$, then we do not necessarily have uniqueness. We will use $P_{n, p}(z ; \mu)$ to denote one such extremal polynomial regardless of whether we have uniqueness or not.

Now, for $p>0$ and a given sequence of measures $\left\{\mu_{k}\right\}_{k=1}^{\infty}$ with compact supports, we can consider the following table of extremal (monic) polynomials with varying measures:

Received June 16, 1994, revised December 5, 1994.

1991 Mathematics Subject Classification. 42C05, 30C15. 


$$
\begin{array}{ccccc}
P_{1, p}\left(z ; \mu_{1}\right) & P_{2, p}\left(z ; \mu_{1}\right) & \cdots & P_{n, p}\left(z ; \mu_{1}\right) & \cdots \\
P_{1, p}\left(z ; \mu_{2}\right) & P_{2, p}\left(z ; \mu_{2}\right) & \cdots & P_{n, p}\left(z ; \mu_{2}\right) & \cdots \\
\vdots & \vdots & & \vdots & \\
P_{1, p}\left(z ; \mu_{k}\right) & P_{2, p}\left(z ; \mu_{k}\right) & \cdots & P_{n, p}\left(z ; \mu_{k}\right) & \cdots \\
\vdots & \vdots & & \vdots &
\end{array}
$$

TABLE 1 . The table of orthogonal polynomials with varying measures.

where we have intentionally omitted entries $P_{0, p}\left(z ; \mu_{k}\right) \equiv 1, k=1,2, \ldots$ So the $k$ th row are extremal polynomials associated with the measure $\mu_{k}$, while the $n$th column corresponds to the sequence of extremal polynomials of degree $n$ with respect to various measures $\mu_{k}, k=1,2, \ldots$ We will concentrate on the asymptotics of the entries in a column of Table 1 when the sequence of measures converges in the weak star topology. Our model case is when $p=2$. It is well known that $P_{n, 2}\left(z ; \mu_{k}\right)$ is the $n$th monic orthogonal polynomial with respect to the measure $\mu_{k}$. So when $p=2$, Table 1 will be referred to as the table of orthogonal polynomials with varying measures. Solutions of these kinds of problems have already been pursued in several concrete situations (cf. $[1,4-10,13,16,17]$, also $\S 3$ below), and different special methods are applied in such solutions. In $\S 2$ we will present a general and simple approach which works for general measures. Our method uses only the extremality of $P_{n, p}$ and the fact that $\mathcal{P}_{n}$ is of finite dimension as a normed linear space. Then in $\S 3$ we use our results in the solutions of some familiar applications as well as some new problems.

\section{General theorems}

Theorem 2.1. Assume $\mu_{k} \stackrel{*}{\longrightarrow} \mu$, and for some compact set $K \subseteq \mathbf{C}, \operatorname{supp}\left(\mu_{k}\right) \subseteq K$. For $n \in \mathcal{N}^{+}$and $p>0$, if $P_{n, p}(z ; \mu)$ exists and is unique, then

$$
\lim _{k \rightarrow \infty} P_{n, p}\left(z ; \mu_{k}\right)=P_{n, p}(z ; \mu),
$$

locally uniformly for $z \in \mathbf{C}$.

The proof of Theorem 2.1 is quite similar to that of the following Theorem 2.2, so we will sketch the proof of Theorem 2.1 after the proof of Theorem 2.2.

Theorem 2.2. Assume $\mu_{k} \stackrel{*}{\longrightarrow} \mu$ and, for some compact set $K \subseteq \mathbf{C}, \operatorname{supp}\left(\mu_{k}\right) \subseteq K$. Assume further that $\mu$ is a discrete measure, i.e., for some $m \in \overline{\mathcal{N}}^{+}$,

$$
\mu=\sum_{j=1}^{m} \alpha_{j} \delta_{\zeta_{j}}, \quad\left(\alpha_{j}>0\right)
$$

where (here and in the sequel) $\delta_{\zeta}$ denotes the unit measure supported at the point $\zeta$. For $n \in \mathcal{N}^{+}$and $p>0$, let $\left\{z_{j, k}=z_{j, k}(n, p)\right\}_{j=1}^{n}$ denote the zeros of $P_{n, p}\left(z ; \mu_{k}\right)$. Then for $n \geq m$ and with suitable arrangement of the zeros of $P_{n, p}\left(z ; \mu_{k}\right)$, we have

$$
\lim _{k \rightarrow \infty} z_{j, k}=\zeta_{j}, \quad j=1,2, \ldots, m .
$$


Proof. Write $P_{n, p}\left(z ; \mu_{k}\right)=\sum_{j=0}^{n} a_{j, k} z^{j}$ (note that $a_{n, k}=1$ ), and let

$$
\tilde{P}_{k}(z):=\frac{P_{n, p}\left(z ; \mu_{k}\right)}{\max _{0 \leq j \leq n}\left|a_{j, k}\right|} .
$$

Then $\left|\tilde{P}_{k}(z)\right| \leq \sum_{j=0}^{n}|z|^{j}$. Thus $\left\{\tilde{P}_{k}\right\}_{k=1}^{\infty} \subseteq \mathcal{P}_{n}$ forms a normal family on C. Let $\tilde{P}(z)$ be a limit function of this family, and let $\Lambda$ be a subsequence of $\mathcal{N}^{+}$such that

$$
\lim _{\substack{k \rightarrow \infty \\ k \in \Lambda}} \tilde{P}_{k}(z)=\tilde{P}(z)
$$

locally uniformly on C. Now

$$
\begin{aligned}
& \left.\left|\int\right| \tilde{P}_{k}(z)\right|^{p} d \mu_{k}-\int|\tilde{P}(z)|^{p} d \mu \mid \\
& \quad \leq\left|\int\left(\left|\tilde{P}_{k}(z)\right|^{p}-|\tilde{P}(z)|^{p}\right) d \mu_{k}\right|+\left.\left|\int\right| \tilde{P}(z)\right|^{p}\left(d \mu_{k}-d \mu\right) \mid \\
& \quad \leq\left.\mu_{k}(K) \max _{z \in K}|| \tilde{P}_{k}(z)\right|^{p}-|\tilde{P}(z)|^{p}|+| \int|\tilde{P}(z)|^{p}\left(d \mu_{k}-d \mu\right) \mid \rightarrow 0
\end{aligned}
$$

as $k \rightarrow \infty$ and $k \in \Lambda$. So

$$
\lim _{\substack{k \rightarrow \infty \\ k \in \Lambda}} \int\left|\tilde{P}_{k}(z)\right|^{p} d \mu_{k}=\int|\tilde{P}(z)|^{p} d \mu .
$$

On the other hand, using the fact that $\max _{0 \leq j \leq n}\left|a_{j, k}\right| \geq 1$ and the extremality of $P_{n, p}$, we have

$$
\int\left|\tilde{P}_{k}(z)\right|^{p} d \mu_{k} \leq \int\left|P_{n, p}\left(z ; \mu_{k}\right)\right|^{p} d \mu_{k} \leq \int\left|z^{n-m} \prod_{j=1}^{m}\left(z-\zeta_{j}\right)\right|^{p} d \mu_{k}
$$

so

$$
\lim _{\substack{k \rightarrow \infty \\ k \in \Lambda}} \int\left|\tilde{P}_{k}(z)\right|^{p} d \mu_{k} \leq \int\left|z^{n-m} \prod_{j=1}^{m}\left(z-\zeta_{j}\right)\right|^{p} d \mu=0 .
$$

This, together with (1), gives

$$
\int|\tilde{P}(z)|^{p} d \mu=0
$$

Thus $\tilde{P}\left(\zeta_{j}\right)=0, j=1,2, \ldots, m$. Now, from the definition of $\tilde{P}_{k}(z)$, we know that at least one of its coefficients is of absolute value 1 . Therefore $\tilde{P}(z)$ has at least one coefficient whose absolute value is equal to 1 , so $\tilde{P}(z) \not \equiv 0$. Hence $\tilde{P}(z)=q(z) \prod_{j=1}^{m}\left(z-\zeta_{j}\right)$ for some $0 \not \equiv q \in \mathcal{P}_{n-m}$. Since $\tilde{P}(z)$ is an arbitrary limit function of the normal family $\left\{\tilde{P}_{k}(z)\right\}_{k=1}^{\infty}$, Hurwitz's theorem now implies the conclusion of the theorem.

We remark that when $p>1$, it is known (cf. [20]) that all zeros of $P_{n, p}\left(\cdot ; \mu_{k}\right)$ are contained in the convex hull of $\operatorname{supp}\left(\mu_{k}\right)$, which, in turn, is contained in the convex hull of $K$ (which is still compact). It then follows that $\left\{P_{n, p}\left(\cdot ; \mu_{k}\right)\right\}_{k=1}^{\infty}$ forms a normal family, so there is no need to introduce $\tilde{P}_{k}$ when $p>1$. 
Proof of Theorem 2.1. From the uniqueness of $P_{n, p}(z ; \mu)$ we know that $\operatorname{supp}(\mu)$ contains at least $n+1$ points. Let $\tilde{P}_{k}(z)$ be defined as in the proof of Theorem 2 . We now prove that $\left\{\max _{0 \leq j \leq n}\left|a_{j, k}\right|\right\}_{k=1}^{\infty}$ is a bounded sequence. Assume, to the contrary, that there is a subsequence $\Lambda$ of $\mathcal{N}^{+}$such that

$$
\lim _{\substack{k \rightarrow \infty \\ k \in \Lambda}} \max _{0 \leq j \leq n}\left|a_{j, k}\right|=\infty .
$$

Then, since $\left\{\tilde{P}_{k}(z)\right\}_{k=1}^{\infty}$ is a normal family, we can find a sequence $\Lambda^{\prime} \subseteq \Lambda$ and $\tilde{P}(z) \in \mathcal{P}_{n}$ with $\tilde{P}(z) \not \equiv 0$ (recall that at least one coefficient of $\tilde{P}(z)$ is of absolute value 1$)$, such that $\lim _{k \rightarrow \infty, k \in \Lambda^{\prime}} \tilde{P}_{k}(z)=\tilde{P}(z)$, locally uniformly on C. As in the proof of Theorem 2.2, we have

$$
\lim _{\substack{k \rightarrow \infty \\ k \in \Lambda^{\prime}}} \int\left|\tilde{P}_{k}(z)\right|^{p} d \mu_{k}=\int|\tilde{P}(z)|^{p} d \mu
$$

But the extremality of $P_{n, p}$ yields

$$
\int\left|\tilde{P}_{k}(z)\right|^{p} d \mu_{k}=\int\left|\frac{P_{n, p}\left(z ; \mu_{k}\right)}{\max _{0 \leq j \leq n}\left|a_{j, k}\right|}\right|^{p} d \mu_{k} \leq \frac{\int|z|^{p n} d \mu_{k}}{\max _{0 \leq j \leq n}\left|a_{j, k}\right|^{p}} .
$$

Letting $k \in \Lambda^{\prime}$ and $k \rightarrow \infty$ in the above relation gives

$$
\int|\tilde{P}(z)|^{p} d \mu \leq \frac{\int|z|^{p n} d \mu}{\lim _{\substack{k \rightarrow \infty \\ k \in \Lambda^{\prime}}} \max _{0 \leq 1 \leq n}\left|a_{j, k}\right|^{p}}=0
$$

which, together with the fact that $\operatorname{supp}(\mu)$ contains at least $n+1$ points, implies $\tilde{P}(z) \equiv 0$, a contradiction. Hence $\left\{a_{j, k}\right\}$ is uniformly bounded. This tells us that the sequence $\left\{P_{n, p}\left(z ; \mu_{k}\right)\right\}_{k=1}^{\infty}$ itself is a normal family on $\mathbf{C}$. Let $P(z)$ be a limit function of this family. All we need to do now is to show that $P(z) \equiv P_{n, p}(z ; \mu)$. This is proved by using the extremality of $P_{n, p}$ as follows.

Take $\Lambda \subseteq \mathcal{N}^{+}$such that $\lim _{\substack{k \in \Lambda \\ \text { (n) }}} P_{n, p}\left(z ; \mu_{k}\right)=P(z)$. Note that

$$
\int\left|P_{n, p}\left(z ; \mu_{k}\right)\right|^{p} d \mu_{k} \leq \int\left|Q_{n}(z)\right|^{p} d \mu_{k}, \quad \text { for any } Q_{n}(z)=z^{n}+\cdots \in \mathcal{P}_{n}
$$

Letting $k \in \Lambda$ and $k \rightarrow \infty$ yields

$$
\int|P(z)|^{p} d \mu \leq \int\left|Q_{n}(z)\right|^{p} d \mu, \quad \text { for any } Q_{n}(z)=z^{n}+\cdots \in \mathcal{P}_{n} .
$$

Then the extremality and the uniqueness of $P_{n, p}(z ; \mu)$ force $P(z) \equiv P_{n, p}(z ; \mu)$. This completes the proof of Theorem 2.1.

\section{Applications}

3.1. The unit circle case. In this subsection, we consider measures supported on the unit circle and apply our general theorems to the so-called Frequency Analysis Problem. 
A (causal) signal is a sequence $x=\{x(m)\}_{m=0}^{\infty}$ of real numbers. Suppose we can observe (or measure) a signal $x$ exactly and suppose we know the signal is of the form

$$
x(m)=\sum_{j=-I}^{I} \alpha_{j} e^{i \omega_{j} m}, \quad m=0,1,2, \ldots,
$$

where $I$ is a (known) positive integer, the (unknown) $\omega_{j}$ 's are called the frequencies of the signal and satisfy $0=\omega_{0}<\omega_{1}<\cdots<\omega_{I}<\pi$ and $\omega_{j}=-\omega_{-j}$, and the (unknown) coefficients $\alpha_{j}$ satisfy $\alpha_{j}=\overline{\alpha_{-j}}$. Then the classical frequency analysis problem is to estimate the frequencies $\omega_{1}, \omega_{2}, \ldots, \omega_{I}$ from the observed $N$-truncated signal $x_{N}=\{x(m)\}_{k=0}^{N-1}$ of signal $x$. There are various methods for solving this problem (cf. $[3,12,14,16])$. Recently, a method developed from ideas of Wiener and Levinson and using the theory of orthogonal polynomials on the unit circle (the socalled Szegö polynomials) has been under investigation in $[4-10,16,17]$. The main method and theory can be summarized as in the following theorem.

Theorem 3.1. Let $X_{N}(z):=\sum_{m=0}^{N-1} x(m) z^{-m}$ and

$$
d \psi_{N}(\theta):=\frac{1}{2 \pi N}\left|X_{N}\left(e^{i \theta}\right)\right|^{2} d \theta, \quad 0 \leq \theta \leq 2 \pi .
$$

For $n \in \mathcal{N}^{+}$, let $\varphi_{n}(z):=\varphi_{n}\left(z ; d \psi_{N}\right)$ be the $n$th monic orthogonal polynomial with respect to the distribution $\psi_{N}$ on the unit circle, i.e., $\varphi_{n}(z)=z^{n}+\cdots \in \mathcal{P}_{n}$ and

$$
\int_{0}^{2 \pi} \varphi_{n}\left(e^{i \theta}\right) e^{-i k \theta} d \psi_{N}(\theta)=0, \quad k=0,1, \ldots, n-1 .
$$

Let $L=0$ if $\alpha_{0}=0$ and $L=1$ if $\alpha_{0} \neq 0$. Then for each fixed $n \geq 2 I+L$, the $2 I+L$ zeros of largest modulus approach the points $e^{i \omega_{j}}, L \leq|j| \leq I$, as $N \rightarrow \infty$, and there exists a constant $K_{n} \in(0,1)$ such that the remaining $n-2 I-L$ zeros are bounded in the disk $|z| \leq K_{n}$.

We will obtain a new proof of Theorem 3.1 as a corollary of the following more general result, Theorem 3.2. We first need to introduce some notations.

Let $\Gamma:=\{z:|z|=1\}$. For a measure $\mu$ on $\Gamma$, let $\mu^{\prime}(\theta)=d \mu\left(e^{i \theta}\right) / d \theta$. We say $\mu$ satisfies Szegö's condition if $\int_{0}^{2 \pi} \log \mu^{\prime}(\theta) / d \theta>-\infty$. When $\mu$ satisfies Szegő's condition, the following so-called Szegö function for $\mu$ is well defined (cf. [21]):

$$
D(z ; \mu):=\exp \left\{\frac{1}{4 \pi} \int_{0}^{2 \pi} \frac{e^{i \theta}+z}{e^{i \theta}-z} \log \mu^{\prime}(\theta) d \theta\right\},
$$

and we also know that

a) $D(z ; \mu)$ is in $\mathbf{H}^{2}$ and non-zero for $|z|<1$,

b) $\left|D\left(e^{i \theta} ; \mu\right)\right|^{2}=\mu^{\prime}(\theta)$ a.e. on $[0,2 \pi]$, and

c) $D(0 ; \mu)>0$.

Theorem 3.2. Let $\mu_{k}$ and $\mu$ be measures on $\Gamma$, and let $\mu_{k} \stackrel{*}{\longrightarrow} \mu$. Assume further that $\mu_{k}$ satisfies Szegö's condition and $\mu$ is a discrete measure of the form $\mu=\sum_{j=1}^{m} \alpha_{j} \delta_{\zeta_{j}}$ with $\left|\zeta_{j}\right|=1, j=1,2, \ldots, m$. Finally, assume that for $j \in \mathcal{N}$ with $|j| \leq m$, there is a constant $C>0$ such that

$$
\left|\int_{\Gamma} e^{i j \theta} d \mu_{k}-\int_{\Gamma} e^{i j \theta} d \mu\right| \leq C D^{2}\left(0 ; \mu_{k}\right), \quad k=1,2,3, \ldots
$$


Then for $n \geq m$, the $m$ zeros of $P_{n, 2}\left(z ; \mu_{k}\right)$ of largest modulus approach the points $\zeta_{j}, j=1,2, \ldots, m$, as $k \rightarrow \infty$, and there exists a constant $K_{n} \in(0,1)$ such that all remaining $n-m$ zeros are bounded in the disk $|z| \leq K_{n}$.

Remark. The quantity $D^{2}(0 ; \mu)$ is just the geometric mean

$$
G(\mu):=\exp \frac{1}{2 \pi} \int_{0}^{2 \pi} \log \mu^{\prime}(\theta) d \theta
$$

of $\mu$, cf. [21, Chap. X].

Proof of Theorem 3.2. By Theorem 2.2, there are $m$ zeros of $P_{n, 2}\left(z ; \mu_{k}\right)$ approaching the points $\zeta_{j}, j=1,2, \ldots, m$, as $k \rightarrow \infty$. Further, from the proof of Theorem 2.1, we know that $\left\{P_{n, 2}\left(z ; \mu_{k}\right)\right\}_{k \geq 1}$ is a normal family and every limit function of this family is of the form $Q(z) \prod_{j=1}^{m}\left(z-\zeta_{j}\right)$ with $Q(z)=z^{n-m}+\cdots \in \mathcal{P}_{n-m}$. Since all zeros of $P_{n, 2}\left(z ; \mu_{k}\right)$ are contained in the open unit disk $|z|<1$ (cf. [21, Theorem 11.4.1]), all zeros of $Q$ must lie in $|z| \leq 1$. To finish the proof, it suffices to show that all zeros of $Q$ are uniformly bounded away from the unit circle $\Gamma$. Let us consider any such $Q$ with $\lim _{k \rightarrow \infty, k \in \Lambda} P_{n, 2}\left(z ; \mu_{k}\right)=Q(z) \prod_{j=1}^{m}\left(z-\zeta_{j}\right)$, for some $\Lambda \subseteq \mathcal{N}^{+}$, locally uniformly for $z \in \mathrm{C}$. From $[2,(1.20)]$, we can derive

$$
\frac{1}{2 \pi} \int_{0}^{2 \pi}\left|\frac{\prod_{j=1}^{m}\left(e^{i \theta}-\zeta_{j}\right)}{P_{n, 2}\left(e^{i \theta} ; \mu_{k}\right)}\right|^{2} d \theta=\frac{\int_{0}^{2 \pi}\left|\prod_{j=1}^{m}\left(e^{i \theta}-\zeta_{j}\right)\right|^{2} d \mu_{k}}{\int_{0}^{2 \pi}\left|P_{n, 2}\left(e^{i \theta} ; \mu_{k}\right)\right|^{2} d \mu_{k}} \quad(n \geq m) .
$$

Now, since $\int_{\Gamma}\left|\Pi\left(e^{i \theta}-\zeta_{j}\right)\right|^{2} d \mu=\sum \alpha_{k} \Pi\left|\left(\zeta_{k}-\zeta_{j}\right)\right|^{2}=0$, by (4) there is a $C>0$ such that

$$
\int_{\Gamma}\left|\prod_{j=1}^{m}\left(e^{i \theta}-\zeta_{j}\right)\right|^{2} d \mu_{k} \leq C D^{2}\left(0 ; \mu_{k}\right)
$$

On the other hand, we have, using properties b), then a), of $D\left(z ; \mu_{k}\right)$,

$$
\begin{aligned}
\int_{\Gamma}\left|P_{n, 2}\left(e^{i \theta} ; \mu_{k}\right)\right|^{2} d \mu_{k} & \geq \int_{0}^{2 \pi}\left|P_{n, 2}\left(e^{i \theta} ; \mu_{k}\right)\right|^{2}\left|D\left(e^{i \theta} ; \mu_{k}\right)\right|^{2} d \theta \\
& =\int_{0}^{2 \pi}\left|P_{n, 2}^{*}\left(e^{i \theta} ; \mu_{k}\right)\right|^{2}\left|D\left(e^{i \theta} ; \mu_{k}\right)\right|^{2} d \theta \\
& \geq 2 \pi D^{2}\left(0 ; \mu_{k}\right) .
\end{aligned}
$$

Here, in the equality, we have used the ${ }^{*}$ operation on a polynomial $p_{n}(z)$ of degree $n: p_{n}^{*}(z):=z^{n} \overline{p_{n}(1 / \bar{z})}$. So equation (5) yields

$$
\frac{1}{2 \pi} \int_{0}^{2 \pi}\left|\frac{\prod_{j=1}^{m}\left(e^{i \theta}-\zeta_{j}\right)}{P_{n, 2}\left(e^{i \theta} ; \mu_{k}\right)}\right|^{2} d \theta \leq \frac{C D^{2}\left(0 ; \mu_{k}\right)}{2 \pi D^{2}\left(0 ; \mu_{k}\right)}=\frac{C}{2 \pi} .
$$

Now letting $k \rightarrow \infty$ and $k \in \Lambda$ gives

$$
\frac{1}{2 \pi} \int_{0}^{2 \pi} \frac{1}{\left|Q\left(e^{i \theta}\right)\right|^{2}} d \theta \leq \frac{C}{2 \pi}
$$

It follows from this that there is a constant $K_{n}^{\prime} \in(0,1)$ depending on $C$ but not on $Q$ such that all zeros of $Q$ are in $|z| \leq K_{n}^{\prime}$. An application of Hurwitz's theorem will then complete the proof. 
Proof of Theorem 3.1. We verify that assumptions of Theorem 3.2 are satisfied. It is known that (cf. [5, Theorem 7])

$$
d \psi_{N}(\theta)=\frac{1}{2 \pi N}\left|X_{N}\left(e^{i \theta}\right)\right|^{2} d \theta \stackrel{*}{\longrightarrow} \sum_{|j| \leq I}\left|\alpha_{j}\right|^{2} d \delta_{e^{i \omega_{j}}}\left(e^{i \theta}\right)=: d \psi(\theta) .
$$

Let $d \mu_{k}(z):=d \psi_{k}(\theta)$ and $d \mu(z):=d \psi(\theta)$ with $z=e^{i \theta}$. Then in order to finish the proof we need to check that (4) holds for every $j \in \mathcal{N}$ with $|j| \leq m$.

First, it is easy to see that

$$
D\left(0 ; d \mu_{k}\right)=D\left(0 ; \frac{1}{2 \pi k}\left|X_{k}\left(e^{i \theta}\right)\right|^{2} d \theta\right) \geq|x(h)| \frac{1}{\sqrt{2 \pi k}},
$$

where $x(h)$ is the first non-zero term in the sequence $\{x(m)\}_{m=0}^{\infty}$. Next, as shown in [10],

$$
\int_{0}^{2 \pi} e^{i j \theta} \frac{1}{2 \pi k}\left|X_{k}\left(e^{i \theta}\right)\right|^{2} d \theta=\int_{0}^{2 \pi} e^{i j \theta} d\left(\sum_{|j| \leq I}\left|\alpha_{j}\right|^{2} \delta_{e^{i \omega_{j}}}\left(e^{i \theta}\right)\right)+\mathcal{O}\left(\frac{1}{k}\right) \quad(k \rightarrow \infty) .
$$

Now it is clear that (4) holds for all $j \in \mathcal{N}$. This completes the proof.

The measure $d \psi_{N}(\theta)$ constructed in Theorem 3.1 (originally introduced in [5]) can be thought as a Fejer type kernel (cf. Theorem 7 and its proof, in [5]). As is known, the Fejer kernel is not the "best" in the sense that the convolution operator formed by the Fejer kernel wouldn't yield Jackson's order of convergence [11, $\S 2.2$ and 4.4]. So it seems there is room for other choices by taking different kernel functions. As an example, let us consider the following kernel $K_{N}(t)$ :

$$
K_{N}(t):=C_{N}\left|\sum_{m=0}^{N-1} a_{m} e^{-i m t}\right|^{2}
$$

where $a_{m}:=\cos (m+1) \pi /(N+1), m=0,1, \ldots, N-1$, and $C_{N}^{-1}:=\sum_{m=0}^{N-1} a_{m}^{2}$. It can be verified (for example, by using an argument similar to that in [11, pp. 75-77, 130-132]) that for a continuously differentiable periodic function $f$,

$$
\frac{1}{2 \pi} \int_{0}^{2 \pi} f(\theta) K_{N}(t-\theta) d \theta=f(t)+\mathcal{O}\left(\omega\left(f^{\prime} ; \frac{1}{N}\right)\right) \quad(N \rightarrow \infty) .
$$

In particular, for every fixed $n \in \mathcal{N}$, we have

$$
\frac{1}{2 \pi} \int_{0}^{2 \pi} e^{i n \theta} K_{N}(t-\theta) d \theta=e^{i n t}+\mathcal{O}\left(\frac{1}{N}\right) \quad(N \rightarrow \infty) .
$$

Now define the following measure on the unit circle:

$$
d \hat{\psi}_{N}(\theta):=\frac{C_{N}}{2 \pi}\left|A_{N}\left(e^{i \theta}\right)\right|^{2} d \theta, \quad N=1,2, \ldots,
$$

where $A_{N}(z):=\sum_{m=0}^{N-1} a_{m} x(m) z^{-m}$. A comparison between $A_{N}(z)$ and $X_{N}(z)$ in Theorem 3.1 reveals that we "window" the original signal $\{x(m)\}_{m=0}^{N-1}$ using $\left\{a_{m}\right\}$ before performing the $z$-transform. With $d \hat{\psi}_{N}(\theta)$, it is easy to obtain the convergence result from Theorem 3.2. We have 
Theorem 3.3. With all previous notations, let $\varphi_{n}(z):=\varphi_{n}\left(z ; d \hat{\psi}_{k}\right)$ be the nth monic orthogonal polynomial with respect to the measure $d \hat{\psi}_{k}$ on the unit circle. Then for each fixed $n \geq 2 I+L$, the $2 I+L$ zeros of the largest modulus approach the point $e^{i \omega_{j}}$, $L \leq|j| \leq I$, as $k \rightarrow \infty$.

Proof. Let $d \mu_{k}(z):=d \hat{\psi}_{k}(\theta)\left(z=e^{i \theta}\right)$ and $d \mu(z):=\sum_{|j| \leq I}\left|\alpha_{j}\right|^{2} d \delta_{e^{i \omega_{j}}}(z)$. We first observe that

$$
\begin{aligned}
D\left(0 ; d \mu_{k}\right) & =D\left(0 ; \frac{C_{k}}{2 \pi}\left|\sum_{m=0}^{k-1} a_{m} x(m) e^{-i m \theta}\right|^{2} d \theta\right) \\
& \geq \sqrt{\frac{C_{k}}{2 \pi}}\left|\cos \left(\frac{h+1}{k+1} \pi\right) \cdot x(h)\right|
\end{aligned}
$$

where $x(h)$ is defined as in the proof of Theorem 3.1.

Next we have, using (3),

$$
\begin{aligned}
\int_{0}^{2 \pi} e^{i n \theta} & \frac{C_{k}}{2 \pi}\left|A_{k}\left(e^{i \theta}\right)\right|^{2} d \theta \\
= & \sum_{j=-I}^{I}\left|\alpha_{j}\right|^{2} \frac{C_{k}}{2 \pi} \int_{0}^{2 \pi} e^{i n \theta}\left|\sum_{m=0}^{k-1} a_{m} e^{i m\left(\omega_{j}-\theta\right)}\right|^{2} d \theta \\
& \quad+\sum_{j \neq l} \alpha_{j} \bar{\alpha}_{l} \sum_{m, q=0}^{k-1} a_{m} a_{q} \frac{C_{k}}{2 \pi} \int_{0}^{2 \pi} e^{i n \theta+i m\left(\omega_{j}-\theta\right)-i q\left(\omega_{l}-\theta\right)} d \theta \\
= & \sum_{j=-I}^{I}\left|\alpha_{j}\right|^{2} e^{i n \omega_{j}}+\mathcal{O}\left(\frac{1}{k}\right)+\left(\sum_{j \neq l} \alpha_{j} \alpha_{-l} e^{i n \omega_{j}}\left\{\sum_{q=0}^{k-1} a_{q+n} a_{q} e^{i q\left(\omega_{j}-\omega_{l}\right)}\right\}\right) C_{k},
\end{aligned}
$$

where in the last equality, we used (7). Writing

$$
a_{m}=\frac{1}{2}\left(e^{i(m+1) \pi /(k+1)}+e^{-i(m+1) \pi /(k+1)}\right)
$$

and summing geometric series in the sum inside the curved brackets above, we can see that the sum in the coefficient of $C_{k}$ is bounded.

Finally, note that $\lim _{k \rightarrow \infty} k C_{k}=\left(\int_{0}^{1} \cos ^{2}(\pi \theta) d \theta\right)^{-1}=2$. Hence

$$
\int_{0}^{2 \pi} e^{i n \theta} \frac{C_{k}}{2 \pi}\left|A_{k}\left(e^{i \theta}\right)\right|^{2} d \theta-\sum_{|j| \leq I}\left|\alpha_{j}\right|^{2} e^{i n \omega_{j}}=\mathcal{O}\left(\frac{1}{k}\right), \quad k \rightarrow \infty,
$$

and (8) yields

$$
D\left(0 ; d \mu_{k}\right) \geq \frac{C}{\sqrt{k}}
$$

for some constant $C>0$ independent of $k$. This, together with (9), verifies that condition (4) holds. So an application of Theorem 3.2 completes the proof of Theorem 3.3.

The new measure $d \hat{\psi}_{N}(\theta)$ gives us the same order of convergence as the old measure $d \psi_{N}(\theta)$ in regard to estimating the frequencies $\omega_{j}$ from the $m$ zeros of the corresponding Szegö polynomials. It would be nice to know whether there exists 
a window function such that the order of convergence in estimating $\omega_{j}$ 's could be improved.

From Theorem 3.1 and its proof we do not know if $\lim _{N \rightarrow \infty} \varphi_{n}\left(z ; d \psi_{N}\right)$ exists when $n>2 I+L$, although we know that every limit function of the normal family $\left\{\varphi_{n}\left(z ; d \psi_{N}\right)\right\}_{N=1}^{\infty}$ can be divided evenly by $\prod_{L \leq|j| \leq I}\left(z-e^{i \omega_{j}}\right)$. Examples are known (cf. $[17,18]$ ) in which $\left\{\varphi_{n}\left(z ; d \psi_{N}\right)\right\}$ does not converge when $n>2 I+L$. In view of these observations, it is interesting that Jones, Njåstad and Waadeland [8] give an alternative way of using Szegö polynomials so that the convergence is always guaranteed. Their result can be stated in our notation as follows.

Let

$$
F_{N}(z):=\int_{0}^{2 \pi} \frac{e^{i \theta}+z}{e^{i \theta}-z} d \psi_{N}(\theta), \quad N=1,2, \ldots
$$

Then for $0<R<1$, the function $F_{N}(R z)$ is analytic and $\operatorname{Re} F_{N}(R z)>0$ for $|z| \leq 1$. Thus the function $F_{N}(R z)$ is a Carathéodory function, cf. [22], so it has the following integral representation (see, for example, [22, Theorem IV.15]) for some measure $d \psi_{N}^{R}(\theta)$ :

$$
F_{N}(R z)=\int_{0}^{2 \pi} \frac{e^{i \theta}+z}{e^{i \theta}-z} d \psi_{N}^{R}(\theta), \quad|z|<1 .
$$

Theorem 3.4. ([8]) The limit

$$
\lim _{R \rightarrow 1^{-}} \lim _{N \rightarrow \infty} \varphi_{n}\left(z ; d \psi_{N}^{R}\right)=\hat{\varphi}_{n}(z)
$$

exists, and for $n \geq 2 I+L$ the polynomial $\hat{\varphi}_{n}(z)$ is of the form

$$
\hat{\varphi}_{n}(z)=\prod_{L \leq|j| \leq I}\left(z-e^{i \omega_{j}}\right) \cdot \prod_{j=1}^{n-2 I-L}\left(z-z_{j}^{(n)}\right),
$$

where

$$
\left|z_{j}^{(n)}\right| \leq 1, \quad j=1, \ldots, n-2 I-L, \quad n=2 I+L+1,2 I+L+2, \ldots .
$$

In [8], the points $z_{j}^{(n)}$ are called the "uninteresting" zeros. Theorem 3.4 tells us that all uninteresting zeros lie on the closed unit disk. As is pointed out in [8], it is desirable to know whether the " $K_{n}$-result" - all uninteresting zeros are contained in a smaller disk with radius $K_{n}(<1)$ - holds as in Theorem 3.1. Using Theorems 2.1 and 3.2 , we establish the " $K_{n}$-result" for Theorem 3.4 :

Theorem 3.5. For each $n>2 I+L$, there exists a constant $K_{n} \in(0,1)$ such that

$$
\left|z_{j}^{(n)}\right| \leq K_{n}, \quad j=1, \ldots, n-2 I-L,
$$

where $z_{j}^{(n)}$ is the uninteresting zero, as defined in Theorem 3.4 .

Proof. By (6), we have, for $|z| \leq 1$,

$$
\lim _{N \rightarrow \infty} F_{N}(R z)=\lim _{N \rightarrow \infty} \int_{0}^{2 \pi} \frac{e^{i \theta}+R z}{e^{i \theta}-R z} d \psi_{N}(\theta)=\int_{0}^{2 \pi} \frac{e^{i \theta}+R z}{e^{i \theta}-R z} d \psi(\theta)=: F(R z) .
$$

Since $F(R z)$ is a Carathéodory function, there is a measure $d \psi^{R}(\theta)$ such that

$$
F(R z)=\int_{0}^{2 \pi} \frac{e^{i \theta}+z}{e^{i \theta}-z} d \psi^{R}(\theta), \quad|z| \leq 1 .
$$


Actually, by the form of $d \psi(\theta)$ in $(6)$, it is easy to show that

$$
d \psi^{R}(\theta)=\frac{1}{2 \pi} \sum_{|j| \leq I}\left|\alpha_{j}\right|^{2} \operatorname{Re}\left(\frac{e^{i \omega_{j}}+\operatorname{Re}^{i \theta}}{e^{i \omega_{j}}-R e^{i \theta}}\right) d \theta .
$$

Note that (11) tells us that

$$
\lim _{N \rightarrow \infty} \int_{0}^{2 \pi} \frac{e^{i \theta}+z}{e^{i \theta}-z} d \psi_{N}^{R}(\theta)=\int_{0}^{2 \pi} \frac{e^{i \theta}+z}{e^{i \theta}-z} d \psi^{R}(\theta), \quad|z| \leq 1 .
$$

It is easy to derive from this that

$$
d \psi_{N}^{R}(\theta) \stackrel{*}{\longrightarrow} d \psi^{R}(\theta) \quad \text { as } \quad N \rightarrow \infty .
$$

By Theorem 2.1, this, together with $\operatorname{supp}\left(d \psi^{R}\right)=[0,2 \pi]$ from (12), implies that

$$
\lim _{N \rightarrow \infty} \varphi_{n}\left(z ; d \psi_{N}^{R}\right)=\varphi_{n}\left(z ; d \psi^{R}\right)
$$

locally uniformly for $z \in \mathbf{C}$. We note that (13) is also established in [8]. Therefore, we have

$$
\lim _{R \rightarrow 1^{-}} \lim _{N \rightarrow \infty} \varphi_{n}\left(z ; d \psi_{N}^{R}\right)=\lim _{R \rightarrow 1^{-}} \varphi_{n}\left(z ; d \psi^{R}\right) .
$$

It is proved in [8] that $\lim _{R \rightarrow 1^{-}} \varphi_{n}\left(z ; d \psi^{R}\right)$ exists, and the limit function is given by (10). Now we will use Theorem 3.2 to show that $\left|z_{j}^{(n)}\right| \leq K_{n}(j=1, \ldots, n-2 I-L)$ for some $K_{n} \in(0,1)$. We need only verify that all hypotheses of Theorem 3.2 are satisfied. It suffices to establish the following three assertions.

(i) There holds $d \psi^{R}(\theta) \stackrel{*}{\longrightarrow} d \psi(\theta)$ as $R \rightarrow 1^{-}$.

(ii) Each measure $d \psi^{R}$ satisfies Szegő's condition, i.e.,

$$
\int_{0}^{2 \pi} \log \left(\psi^{R}\right)^{\prime}(\theta) d \theta>-\infty
$$

(iii) For each $n \in \mathcal{N}$, there is a constant $C_{n}>0$ such that

$$
\left|\int_{0}^{2 \pi} e^{i n \theta} d \psi^{R}(\theta)-\int_{0}^{2 \pi} e^{i n \theta} d \psi(\theta)\right| \leq C_{n} D^{2}\left(0 ; d \psi^{R}\right) .
$$

Assertion (i) follows directly from the fact that

$$
\lim _{R \rightarrow 1^{-}} F(R z)=F(z), \quad|z|<1 .
$$

Now note that

$$
\left|\int_{0}^{2 \pi} e^{i n \theta} d \psi^{R}(\theta)-\int_{0}^{2 \pi} e^{i n \theta} d \psi(\theta)\right|=\left(1-R^{|n|}\right)\left|\int_{0}^{2 \pi} e^{i n \theta} d \psi(\theta)\right|,
$$

so assertions (ii) and (iii) will follow if we can show that

$$
D^{2}\left(0 ; d \psi^{R}\right) \geq \frac{1-R}{1+R} \frac{1}{2 \pi} \sum_{|j| \leq I}\left|\alpha_{j}\right|^{2}
$$


We have, using (12),

$$
\begin{aligned}
D^{2}\left(0 ; d \psi^{R}\right) & =\exp \left\{\frac{1}{2 \pi} \int_{0}^{2 \pi} \log \left(\psi^{R}\right)^{\prime}(\theta) d \theta\right\} \\
& =\exp \left\{\frac{1}{2 \pi} \int_{0}^{2 \pi} \log \left[\frac{1}{2 \pi} \sum_{|j| \leq I}\left|\alpha_{j}\right|^{2} \operatorname{Re}\left(\frac{e^{i \omega_{j}}+R e^{i \theta}}{e^{i \omega_{j}}-\cdot R e^{i \theta}}\right)\right] d \theta\right\} \\
& \geq \exp \left\{\frac{1}{2 \pi} \int_{0}^{2 \pi} \log \left(\frac{1-R}{1+R} \frac{1}{2 \pi} \sum_{|j| \leq I}\left|\alpha_{j}\right|^{2}\right) d \theta\right\} \\
& =\frac{1-R}{1+R} \frac{1}{2 \pi} \sum_{L \leq|j| \leq I}\left|\alpha_{j}\right|^{2} .
\end{aligned}
$$

This establishes (14).

We finish this subsection by proving a result which is a kind of converse of Theorem 2.1 for the case when $p=2$ and $K=\Gamma$.

Theorem 3.6. If $\mu_{k}$ and $\mu$ are unit measures with infinite support sets on $\Gamma$ and if there is a constant $N>0$ such that

$$
\lim _{k \rightarrow \infty} P_{n, 2}\left(z ; \mu_{k}\right)=P_{n, 2}(z ; \mu)
$$

for $z \in \mathbf{C}$ and $n \geq N$, then $\mu_{k} \stackrel{*}{\longrightarrow} \mu$, as $n \rightarrow \infty$.

Remark. Generally, the converse of Theorem 2.1 does not hold. See $\S 3.2$.

Proof. Since $\left\{\mu_{k}\right\}$ is compact in the weak star topology, let $\hat{\mu}$ be a limit with $\mu_{k} \stackrel{*}{\rightarrow} \hat{\mu}$ as $k \rightarrow \infty$ and $k \in \Lambda$, for some $\Lambda \subseteq \mathcal{N}^{+}$. Then, by Theorem 2.1,

$$
\lim _{\substack{k \rightarrow \infty \\ k \in \Lambda}} P_{n, 2}\left(z ; \mu_{k}\right)=P_{n, 2}(z ; \hat{\mu})
$$

and

$$
\lim _{\substack{k \rightarrow \infty \\ k \in \Lambda}} \int_{\Gamma}\left|P_{n, 2}\left(z ; \mu_{k}\right)\right|^{2} d \mu_{k}=\int_{\Gamma}\left|P_{n, 2}(z ; \hat{\mu})\right|^{2} d \hat{\mu} .
$$

So, using the hypothesis, we get

$$
P_{n, 2}(z ; \mu)=P_{n, 2}(z ; \hat{\mu}) \quad \text { for } n \geq N .
$$

Now let $\Phi_{n}(z ; \nu)$ denote the $n$th orthonormal polynomial associated with a unit measure $\nu$ on $\Gamma$, then

$$
\Phi_{n}(z ; \nu)=\frac{P_{n, 2}(z ; \nu)}{\left(\int_{\Gamma}\left|P_{n, 2}(z ; \nu)\right|^{2} d \nu\right)^{1 / 2}} .
$$

It is well known (cf. $[15,19])$ that $\left|\Phi_{n}(z ; \nu)\right|^{-2} d \theta /(2 \pi) \stackrel{*}{\longrightarrow} \nu$ as $n \rightarrow \infty$. Thus,

$$
\frac{1}{\left|\Phi_{n}(z ; \mu)\right|^{2}} \frac{d \theta}{2 \pi} \stackrel{*}{\longrightarrow} \mu, \quad \text { as } n \rightarrow \infty,
$$


and at the same time,

$$
\begin{aligned}
\frac{1}{\left|\Phi_{n}(z ; \mu)\right|^{2}} \frac{d \theta}{2 \pi} & =\frac{\int_{\Gamma}\left|P_{n, 2}(z ; \mu)\right|^{2} d \mu}{\left|P_{n, 2}(z ; \mu)\right|^{2}} \frac{d \theta}{2 \pi}=\frac{\int_{\Gamma}\left|P_{n, 2}(z ; \hat{\mu})\right|^{2} d \hat{\mu}}{\left|P_{n, 2}(z ; \hat{\mu})\right|^{2}} \frac{d \theta}{2 \pi} \\
& =\frac{1}{\left|\Phi_{n}(z ; \hat{\mu})\right|^{2}} \frac{d \theta}{2 \pi} \stackrel{*}{\longrightarrow} \hat{\mu}
\end{aligned}
$$

as $n \rightarrow \infty$, so $\hat{\mu}=\mu$. Since $\hat{\mu}$ is an arbitrary limit of $\left\{\mu_{k}\right\}$, the whole sequence $\left\{\mu_{k}\right\}$ must converge to $\mu$ in the weak star topology.

3.2. The real line case. In this subsection we will show that, in general, the converse of Theorem 2.1 is not true and illustrate how the method used in the proof of Theorems 2.1 and 2.2 can be employed in the situation when the supports of the measures are not compact.

Let $w(x)$ be a non-negative piecewise continuous function defined on $\mathbf{R}, p>1$, and

$$
\int_{-\infty}^{\infty}|x|^{n p} w(x) d x<\infty, \quad n=0,1, \ldots
$$

Examples of such $w(x)$ could be $w(x)=e^{-|x|^{\alpha}}, \alpha>1$. Assume $x_{j} \in \mathbf{R}$ and $w\left(x_{j}\right)>0$ for $j=1,2, \ldots, m$. Denote

$$
q_{0}(x):=\prod_{j=1}^{m}\left(x-x_{j}\right)
$$

Theorem 3.7. Assume $p>1, q_{\epsilon}(x) \in \mathcal{P}_{m}$ for $\epsilon>0$, and $\lim _{\epsilon \rightarrow 0} q_{\epsilon}(x)=q_{0}(x)$, locally uniformly for $x \in \mathbf{R}$. Let $d \mu_{\epsilon}:=w(x) d x /\left|q_{\epsilon}(x)\right|^{p}$ and $d \mu:=w(x) d x /\left|q_{0}(x)\right|^{p}$. Then for $n \geq m$,

$$
\lim _{\epsilon \rightarrow 0} P_{n, p}\left(x ; \mu_{\epsilon}\right)=q_{0}(x) P_{n-m, p}(x ; w(x) d x)=P_{n, p}(x ; \mu),
$$

locally uniformly on $\mathbf{C}$.

Proof. We cannot apply Theorem 2.1 directly since the support of $\mu_{\epsilon}$ may not be compact. But the method used in the proof of Theorem 2.1 can almost be repeated.

Let $P_{n, p}\left(x ; \mu_{\epsilon}\right)=\sum_{j=0}^{n} a_{j, \epsilon} x^{j}$, and let $\tilde{P}_{\epsilon}(x)=P_{n, p}\left(x ; \mu_{\epsilon}\right) / \max _{0 \leq j \leq n}\left|a_{j, \epsilon}\right|$. Then $\left\{\tilde{P}_{\epsilon}(x)\right\}_{\epsilon>0}$ is a normal family on C. We now prove that $\left\{a_{j, \epsilon}\right\}_{j=0, \epsilon>0}^{n}$ is a bounded sequence. Assume, to the contrary, that there is a sequence $\Lambda$ of positive numbers tending to 0 such that

$$
\lim _{\substack{\epsilon \rightarrow 0 \\ \epsilon \in \Lambda}} \max _{0 \leq j \leq n}\left|a_{j, \epsilon}\right|=\infty
$$

Then, since $\left\{\tilde{P}_{\epsilon}(x)\right\}_{\epsilon>0}$ is a normal family, as in the proof of Theorem 2.1, we can find a sequence $\Lambda^{\prime} \subseteq \Lambda$ and $\tilde{P}(x) \in \mathcal{P}_{n}$ with $\tilde{P}(x) \not \equiv 0$ such that $\lim _{\epsilon \rightarrow 0, \epsilon \in \Lambda^{\prime}} \tilde{P}_{\epsilon}(x)=\tilde{P}(x)$, locally uniformly on C. So for $0<\delta<\Delta<\infty$,

$$
\lim _{\substack{\epsilon \rightarrow 0 \\ \epsilon \in \Lambda^{\prime}}} \int_{\delta \leq\left|q_{0}(x)\right| \leq \Delta}\left|\tilde{P}_{\epsilon}(x)\right|^{p} d \mu_{\epsilon}=\int_{\delta \leq\left|q_{0}(x)\right| \leq \Delta}|\tilde{P}(x)|^{p} d \mu .
$$


Now

$$
\begin{aligned}
\int_{\delta \leq\left|q_{0}(x)\right| \leq \Delta}\left|\tilde{P}_{\epsilon}(x)\right|^{p} d \mu_{\epsilon} & \leq \int_{-\infty}^{\infty}\left|\tilde{P}_{\epsilon}(x)\right|^{p} d \mu_{\epsilon} \\
& =\frac{\int_{-\infty}^{\infty}\left|P_{n, p}\left(x ; \mu_{\epsilon}\right)\right|^{p} d \mu_{\epsilon}}{\max _{0 \leq j \leq n}\left|a_{j, \epsilon}\right|^{p}} \leq \frac{\int_{-\infty}^{\infty}\left|x^{n-m} q_{\epsilon}(x)\right|^{p} d \mu_{\epsilon}}{\max _{0 \leq j \leq n}\left|a_{j, \epsilon}\right|^{p}} \\
& =\frac{\int_{-\infty}^{\infty}|x|^{(n-m) p} w(x) d x}{\max _{0 \leq j \leq n}\left|a_{j, \epsilon}\right|^{p}}
\end{aligned}
$$

Letting $\epsilon \rightarrow 0$ with $\epsilon \in \Lambda^{\prime}$ and using (17) yield

$$
\int_{\delta \leq\left|q_{0}(x)\right| \leq \Delta}|\tilde{P}(x)|^{p} d \mu \leq \frac{\int_{-\infty}^{\infty}|x|^{(n-m) p} w(x) d x}{\lim _{\substack{\epsilon \rightarrow 0 \\ \epsilon \in \Lambda^{\prime}}} \max _{0 \leq 1 \leq n}\left|a_{j, \epsilon}\right|^{p}}=0
$$

which implies $\tilde{P}(x) \equiv 0$, a contradiction. Hence $a_{j, \epsilon}$ is uniformly bounded. So the sequence $\left\{P_{n, p}\left(x ; \mu_{\epsilon}\right)\right\}_{\epsilon>0}$ is a normal family. Let $P(x)$ be a limit function of this family.

Take a sequence $\Lambda$ of positive numbers tending to 0 in such a way that $\lim _{\epsilon \in \Lambda} P_{n, p}\left(x ; \mu_{\epsilon}\right)=P(x)$, locally uniformly on C. Note that, for $0<\delta<\Delta<\infty$,

$$
\begin{aligned}
\int_{\delta \leq\left|q_{0}(x)\right| \leq \Delta}|P(x)|^{p} d \mu & =\lim _{\substack{\epsilon \rightarrow 0 \\
\epsilon \in \Lambda}} \int_{\delta \leq\left|q_{0}(x)\right| \leq \Delta}\left|P_{n, p}\left(x ; \mu_{\epsilon}\right)\right|^{p} d \mu_{\epsilon} \\
& \leq \limsup _{\substack{\epsilon \rightarrow 0 \\
\epsilon \in \Lambda}} \int_{-\infty}^{\infty}\left|P_{n, p}\left(x ; \mu_{\epsilon}\right)\right|^{p} d \mu_{\epsilon} \\
& \leq \limsup _{\substack{\epsilon \rightarrow 0 \\
\epsilon \in \Lambda}}^{\infty}\left|Q_{n-m}(x) q_{\epsilon}(x)\right|^{p} d \mu_{\epsilon} \\
& =\int_{-\infty}^{\infty}\left|Q_{n-m}(x)\right|^{p} w(x) d x,
\end{aligned}
$$

for any $Q_{n-m}(x)=x^{n-m}+\cdots \in \mathcal{P}_{n-m}$. This, in particular, gives

$$
\int_{-\infty}^{\infty} \frac{|P(x)|^{p} w(x)}{\left|q_{0}(x)\right|^{p}} d x \leq \int_{-\infty}^{\infty}|x|^{(n-m) p} w(x) d x<\infty .
$$

Since $p>1,|P(x)|^{p} w(x)$ must vanish whenever $q_{0}(x)$ does. This and the assumption that $w\left(x_{j}\right) \neq 0$ imply that $P\left(x_{j}\right)=0$ for $j=1,2, \ldots, m$, so $P(x)=q_{0}(x) R(x)$ for some $R(x)=x^{n-m}+\cdots \in \mathcal{P}_{n-m}$. Using this expression in (18), we get

$$
\int_{-\infty}^{\infty}|R(x)|^{p} w(x) d x \leq \int_{-\infty}^{\infty}\left|Q_{n-m}(x)\right|^{p} w(x) d x
$$

for any $Q_{n-m}(x)=x^{n-m}+\cdots$. Thus, by the uniqueness of the $(n-m)$ th monic extremal $L_{p}(w(x) d x)(p>1)$ polynomial, we must have $R(x)=P_{n-m, p}(x ; w(x) d x)$. Hence, $P(x)=q_{0}(x) P_{n-m, p}(x ; w(x) d x)$. This implies that $\left\{P_{n, p}\left(x ; \mu_{\epsilon}\right)\right\}_{\epsilon>0}$ has only one limit as $\epsilon \rightarrow 0$, so

$$
\lim _{\epsilon \rightarrow 0} P_{n, p}\left(x ; \mu_{\epsilon}\right)=q_{0}(x) P_{n-m, p}(x ; w(x) d x),
$$


which proves the first equality in (16).

The second equality in (16) is based on the fact that any $P_{n}(x) \in \mathcal{P}_{n}$ with $q_{0}(x) \nmid P_{n}(x)$ must satisfy $\int_{-\infty}^{\infty}\left|P_{n}(x)\right|^{p} d \mu=\infty$, because of the singularity at the zeros of $q_{0}(x)$. So, in particular, $q_{0}(x) \mid P_{n, p}(x ; \mu)$, and it then follows easily (as above) that $P_{n, p}(x ; \mu)=q_{0}(x) P_{n-m, p}(x ; w(x) d x)$.

In Theorem 3.5, choose $w(x)$ so that $\operatorname{supp}(w)$ is compact, say $\operatorname{supp}(w)=[-1,1]$. Then we get an example in which, for $n \geq m$,

$$
\lim _{\epsilon \rightarrow 0} P_{n, p}\left(x ; \mu_{\epsilon}\right)=P_{n, p}(x ; \mu),
$$

but, as can easily be verified, $\mu_{\epsilon}$ does not converge to $\mu$. Therefore the converse of Theorem 2.1 does not always hold.

On taking $q_{\epsilon}(x)=\prod_{j=1}^{\sigma}\left[\left(x-x_{j}\right)^{2}+\epsilon^{2}\right]$, we can get the following interesting proposition which is used (when $p=2$ ) in [1] to construct interpolatory quadratures with prescribed node distribution. We remark that the proof in [1] relies on a result on polynomials of $\mathrm{H}$. Cartan.

Corollary 3.8. Let $p>1$. We have, for $n \geq 2 \sigma$,

$$
\lim _{\epsilon \rightarrow 0} P_{n, p}\left(x ; \frac{w(x) d x}{\prod_{j=1}^{\sigma}\left[\left(x-x_{j}\right)^{2}+\epsilon^{2}\right]^{p}}\right)=\prod_{j=1}^{\sigma}\left(x-x_{j}\right)^{2} P_{n-2 \sigma, p}(x ; w(x) d x),
$$

locally uniformly in $\mathbf{C}$.

\section{References}

1. T. Bloom, D. S. Lubinsky, and H. Stahl, Distribution of points for convergent interpolatory integration rules on $(-\infty, \infty)$, Constr. Approx., 9 (1993), 59-82.

2. Ya. L. Geronimus, Orthogonal Polynomials, Consultants Bureau, New York, 1961.

3. F. B. Hildebrand, Introduction to Numerical Analysis, McGraw-Hill, New York, 1965.

4. W. B. Jones and O. Njåstad, Application of the Szegö polynomials to digital signal processing, Rocky Mountain J. Math., 21 (1991), 387-436.

5. W. B. Jones, O. Njåstad, and E. B. Saff, Szegö polynomials associated with Wiener-Levinson filters, J. Comp. Appl. Math., 32 (1990), 387-406.

6. W. B. Jones, O. Njåstad, and H. Waadeland, Asymptotics for Szegö polynomial zeros, Numerical Algorithms, 3 (1992), 255-264.

7. __ Application of Szegő polynomials to frequency analysis, SIAM J. Math. Anal., 25 (1994), 491-512.

8. __ An alternative way of using Szegö polynomials in frequency analysis, in Continued Fractions and Orthogonal Functions, S. Clement Cooper and W. J. Thron, eds., Marcel Dekker, New York, 1994, 141-152.

9. W. B. Jones and E. B. Saff, Szegö polynomials and frequency analysis, in Approximation Theory, G. Anastassiou, ed., Marcel Dekker, New York, 1992, 341-352.

10. W. B. Jones, O. Njåstad, W. J. Thron, and H. Waadeland, Szegő polynomials applied to frequency analysis, J. Comp. Appl. Math., 46 (1993), 217-228.

11. P. P. Korovkin, Linear Operators and Approximation Theory, Hindustan, Delhi, 1960.

12. R. Kumaresam, L. L. Scharf, and A. K. Shaw, An algorithm for pole-zero modeling and spectral analysis, IEEE Trans. ASSP, 34 (1986), 637-640.

13. $\mathrm{X} . \mathrm{Li}$, Remarks on orthogonal polynomials with respect to varying measures and related problems, Internat. J. Math. \& Math. Sci., 2 (1993), 255-258.

14. J. D. Markel and A. N. Gray, Jr., Linear Prediction of Speech, Springer-Verlag, New York, 1976.

15. A. Mate, P. Nevai, and V. Totik, Asymptotics for the ratio of leading coefficients of orthonormal polynomials on the unit circle, Constr. Approx., 1 (1985), 63-69.

16. K. Pan, Asymptotics for Szegö polynomials associated with Wiener-Levinson filters, submitted.

17. K. Pan and E. B. Saff, Asymptotics for zeros of the Szegö polynomials associated with trigonometric polynomial signals, J. Approx. Theory, 71 (1992), 239-251. 
18. A. K. Paul, Anharmonic frequency analysis, Math. Comp., 26 (1972), 437-447.

19. E. Rahmanov, Asymptotics of the ratio of orthogonal polynomials, Math. USSR Sbornik, 32 (1977), 199-213.

20. E. B. Saff, Orthogonal polynomials from a complex perspective, In: Orthogonal Polynomials: Theory and Practice, P. Nevai, ed., Kluwer, Dordrecht, 1990, 363-393.

21. G. Szegö, Orthogonal Polynomials, Amer. Math. Soc. Colloq. Pub., XXIII, New York, 1959.

22. M. Tsuji, Potential Theory in Modern Function Theory, Chelsea, New York, 1959.

University of Central Florida, Department of Mathematics, Orlando, FL 32816, U.S.A. 